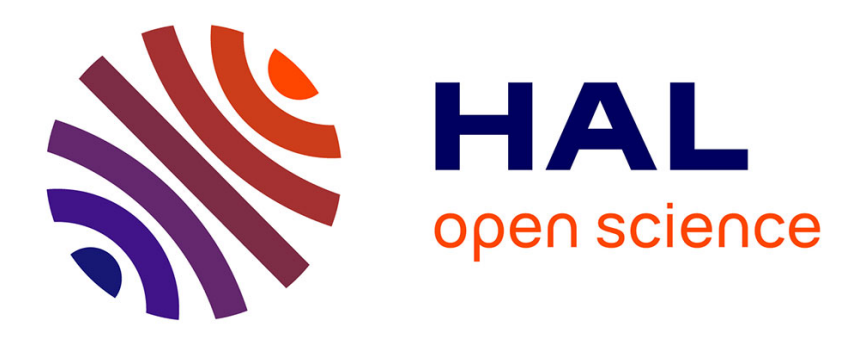

\title{
Method of Monotone Solutions for Reaction-Diffusion Equations
}

\author{
Vitaly Volpert, Vitali Vougalter
}

\section{To cite this version:}

Vitaly Volpert, Vitali Vougalter. Method of Monotone Solutions for Reaction-Diffusion Equations. Journal of Mathematical Sciences, 2021, 253, pp.660 - 675. 10.1007/s10958-021-05260-2 . hal03398980

\section{HAL Id: hal-03398980 \\ https://hal.science/hal-03398980}

Submitted on 26 Oct 2021

HAL is a multi-disciplinary open access archive for the deposit and dissemination of scientific research documents, whether they are published or not. The documents may come from teaching and research institutions in France or abroad, or from public or private research centers.
L'archive ouverte pluridisciplinaire HAL, est destinée au dépôt et à la diffusion de documents scientifiques de niveau recherche, publiés ou non, émanant des établissements d'enseignement et de recherche français ou étrangers, des laboratoires publics ou privés. 


\title{
Method of monotone solutions for reaction-diffusion equations
}

\author{
V. Volpert ${ }^{1,2,3}$, V. Vougalter ${ }^{4}$ \\ ${ }^{1}$ Institut Camille Jordan, UMR 5208 CNRS, University Lyon 1, 69622 Villeurbanne, France \\ ${ }^{2}$ INRIA Team Dracula, INRIA Lyon La Doua, 69603 Villeurbanne, France \\ ${ }^{3}$ RUDN University, ul. Miklukho-Maklaya 6, Moscow, Russia 117198 \\ ${ }^{4}$ Department of Mathematics, University of Toronto, Toronto, M5S 2E4 Ontario, Canada
}

\begin{abstract}
Existence of solutions of reaction-diffusion systems of equations in unbounded domains is studied by the Leray-Schauder (LS) method based on the topological degree for elliptic operators in unbounded domains and on a priori estimates of solutions in weighted spaces. We identify some reaction-diffusion systems for which there exist two sub-classes of solutions separated in the function space, monotone and non-monotone solutions. A priori estimates and existence of solutions are obtained for monotone solutions allowing to prove their existence by the LS method. Various applications of this method are given.
\end{abstract}

AMS subject classification: 35K57, 35J61, 47H11

\section{Introduction}

In this work we present a method to study the existence of solutions of reaction-diffusion equations in unbounded domains. Consider the system of equations

$$
\frac{\partial u}{\partial t}=D \frac{\partial^{2} u}{\partial x^{2}}+F(u)
$$

where $u=\left(u_{1}, \ldots, u_{n}\right), F=\left(F_{1}, \ldots, F_{n}\right), D$ is a diagonal matrix with positive diagonal elements $d_{i}$. A travelling wave solution of this system is a solution $u(x, t)=w(x-c t)$, which satisfies the second-order equation

$$
D w^{\prime \prime}+c w^{\prime}+F(w)=0,
$$

where $c$ is an unknown constant, the wave speed, and $x \in \mathbb{R}$. We will look for solutions with some limits at infinity,

$$
w( \pm \infty)=w_{ \pm}
$$


where $F\left(w_{ \pm}\right)=0$. If $c=0$, then we have a stationary solution of equation (1.1) sometimes also called a standing wave. If, in addition, $w_{+}=w_{-}$, then such solution is called a stationary pulse assuming that it is not identically constant.

In studying the existence of solutions of problem (1.2), (1.3) we will use a modification of the Leray-Schauder (LS) method. In its classical formulation [34], the LS method uses the topological degree for elliptic problems in bounded domains and a priori estimates of solutions. If we consider unbounded domains, then the degree construction and a priori estimates of solutions become different. We use the degree for Fredholm and proper operators with the zero index considered in some special weighted space. A priori estimates of solutions in these spaces are essentially different in comparison with the classical estimates, and in general they do not hold. We will identify some classes of elliptic problems for which there are two types of solutions. A priori estimates are obtained only for one of these types. In order to apply the LS method for these solutions we show that they are separated from another type of solutions. Thus, this construction implies several steps presented below.

Fredholm property for linear elliptic problems. Classical results on linear elliptic problems in bounded domains with a sufficiently smooth boundary affirm that they satisfy the Fredholm property if and only if the ellipticity condition, proper ellipticity and the Lopatinsky condition are satisfied $[1,2,50,51]$. In the case of unbounded domains, one more condition on the invertibility of limiting operators should be imposed [40, 41, 43, 61, 54]. It ensures that the essential spectrum does not cross the origin. In some cases, the index of the operator can be calculated $[17,54]$. The degree construction implies that the essential spectrum lies in the left-half plane of the complex plane [65]. In this case the index of the operator equals zero.

Solvability conditions can be obtained for some linear elliptic operators in unbounded domains without Fredholm property [66, 67]. However, they can be applied to study only some special types of nonlinear operators [68], and the degree theory does not exist in this case.

Some applications of the method of monotone solutions presented in this work concern nonlocal and delay reaction-diffusion equations. Their investigation is based on the Fredholm property of the corresponding elliptic problems [3, 4]. Solvability conditions for various elliptic functional differential equations are studied in [38, 39, 44, 45, 46, 48].

Properness of elliptic problems in unbounded domains. Let us recall that the operator is called proper on closed bounded sets if the intersection of the inverse image of any compact set with any bounded closed set is compact. An important property of proper operators is that the set of solutions of the operator equation (an inverse image of the set 0 ) is compact. Compactness of the set of solutions plays an important role in the degree construction.

It appears that elliptic problems in unbounded domains do not satisfy, in general, this property. We illustrate this situation with the following example. Consider the equation $w^{\prime \prime}+F(w)=0$ on the whole axis, where $F(w)=-w+w^{2}$. It can be easily verified that 
it has a positive solution $w(x)$ with zero limits at infinity. Since the solution is invariant with respect to translation in space, the shifted functions $w(x+h)$ are also solutions of this equation for any $h \in \mathbb{R}$. Therefore, the set of solutions is not compact in conventional Hölder or Sobolev spaces, and the corresponding operator is not proper.

If the invariance of solutions (at infinity) is excluded, then the operators become proper [42]. However, for many elliptic problems, including those considered in this work, invariance with respect to translation is their intrinsic property, and it cannot be excluded. It appears that elliptic operators in unbounded domains become proper in appropriate weighted spaces $[65,54]$. Consider the previous example and the weighted Hölder space $C_{\mu}^{2+\alpha}(\mathbb{R})$, where the weight function $\mu(x)=1+x^{2}$ grows at infinity, and the norm is given by the equality $\|w(\cdot+h)\|_{C_{\mu}^{2+\alpha}(\mathbb{R})}=\|w(\cdot+h) \mu\|_{C^{2+\alpha}(\mathbb{R})}$. Then in any bounded closed set $D$ of the weighted space, there is only a finite interval of values $h$ for which solutions $w(x+h)$ belong to this set (since the norm tends to infinity as $|h|$ grows). Therefore, the set of solutions becomes compact in $D$. This examples illustrates why the introduction of weighted spaces makes the operators proper. As a consequence, topological degree can be constructed in the weighted spaces.

Topological degree for elliptic problems in unbounded domains. The Leray-Schauder degree [34] is applicable for elliptic problems in bounded domains. Since the inverse of the Laplace operator is compact, in this case elliptic operators are reduced to the identity operator plus a compact operator. This construction cannot be used for elliptic operators in unbounded domains since the inverse of the Laplace operator is not compact any more.

Degree construction for Fredholm and proper operators with the zero index is appropriate for elliptic problems in unbounded domains. The first degree construction for Fredholm and proper operators is due to Caccioppoli (see the bibliography in [37]) who defined the degree modulus 2. The important development of this theory was due to the work by Smale [47] who generalized Sard's lemma for Fredholm operators and defined the degree as the number of solutions of the operator equation $f(x)=y$ modulus 2. For almost all $y$ these solutions are regular and their number is finite. Based on the results by Smale, Elworthy and Tromba [20], [21] defined the oriented degree for Fredholm and proper operators of the zero index which homotopy invariant modulus 2 .

Degree construction for Fredholm and proper mappings in [16], [10], [11], [26]-[30] is based on the notions of orientation. Another approach to define the orientation is suggested in [23], [18], [33]. Assuming that the operator $L+\lambda I$ satisfies the Fredholm property for all real $\lambda \geq 0$ and that it has only a finite number $\nu$ of positive eigenvalues (together with their multiplicities), we can define the orientation as $(-1)^{\nu}$. This construction is well adapted for elliptic boundary value problems because it is naturally related to the spectrum of the linearized operator. Similar to other degree constructions, this one requires a precise specification of operators and function spaces [64], [65].

Thus, the topological degree is constructed for general elliptic problems in unbounded domains in weighted spaces $[54,65]$. The application of the LS method requires a priori estimates of solutions in these spaces. 
Method of monotone solutions. A priori estimates of solutions in weighted spaces are quite different in comparison with conventional spaces without weight. The latter are provided by certain regularity of solutions in the case of Hölder spaces and by their decay rate at infinity in the case of Sobolev space. The family of solutions $w(x+h)$ in the example considered above is uniformly bounded in such spaces but not in the weighted spaces. Hence, the introduction of weighted spaces allows the construction of topological degree but it imposes some additional requirements in order to obtain a priori estimates of solutions.

Let us illustrate the situation with the estimates of solutions in the weighted spaces with the following example. Consider the problem (1.2), (1.3) and the corresponding first-order system of equations

$$
w^{\prime}=p, \quad D p^{\prime}=-c p-F_{\tau}(w)
$$

which depends on parameter $\tau$. We look for a trajectory $\gamma$ connecting the stationary points $\left(w_{-}, 0\right)$ and $\left(w_{+}, 0\right)$. If these points are hyperbolic, then in some their small neighborhoods $V_{ \pm}$the trajectory approaches these stationary points exponentially. Hence, the solution $w(x)$ admits a priori estimates in weighted spaces with a polynomial weight $\mu(x)$. In order to estimate the solution on the whole axis uniformly in $\tau$, we need to estimate the length of the interval $L_{\tau}$ where the trajectory is located outside the neighborhoods $V_{+}$and $V_{-}$. It is possible that $L_{\tau} \rightarrow \infty$ as $\tau \rightarrow \tau_{0}$ for some $\tau_{0}$ resulting in the loss of a priori estimates. In general, $L_{\tau}$ is not necessarily bounded, so that a trajectory connecting two stationary points may not exist.

It appears that there are some classes of problems for which it is possible to obtain uniform estimates of $L_{\tau}$. These estimates can be obtained only for some types of solutions and not for all solutions. This means that there are two types of solutions, type 1 and type 2 such that if a solution belongs to a certain type, then it cannot change it during a continuous deformation. A priori estimates of the type 1 solutions (but not of the type 2) allow us to apply the LS method and to prove the existence of solutions.

The two types of solutions are monotone and non-monotone solutions (as functions of $x$ ). There are some classes of problems for which they are separated in the sense specified above, and monotone solutions admit a priori estimates. In a more general setting, the type 1 solutions are not necessarily monotone but they possess some monotonicity properties (for example, a single maximum).

The fact that solutions can preserve their monotonicity in the process of continuous deformation was first noticed in [32]. This method was more systematically developed in $[58,62]$. Some recent applications determine its further development. In this work we present the method and the existence results obtained with it. 


\section{Operators and spaces}

For the functional setting let us introduce the Hölder space $C^{k+\alpha}(\mathbb{R})$ consisting of vectorfunctions of class $C^{k}$, which are continuous and bounded on the axis $\mathbb{R}$ together with their derivatives of order $k$, and such that the derivatives of order $k$ satisfy the Hölder condition with the exponent $\alpha \in(0,1)$. The norm in this space is the usual Hölder norm. Set $E^{1}=C^{2+\alpha}(\mathbb{R}), E^{2}=C^{\alpha}(\mathbb{R})$. Next, we introduce the weighted spaces $E_{\mu}^{1}$ and $E_{\mu}^{2}$ with $\mu(x)=\sqrt{1+x^{2}}$. These spaces are equipped with the norms:

$$
\|w\|_{E_{\mu}^{i}}=\|w \mu\|_{E^{i}}, \quad i=1,2 .
$$

Following $[58,62]$ we introduce the operators which will allow us to study travelling waves, that is solutions of problem (1.2), (1.3). Consider an infinitely differentiable vector-function $\eta(x)$ such that

$$
\eta(x)=\left\{\begin{array}{ll}
w_{-} & , \quad x \leq-1 \\
w_{+} & , \quad x \geq 1
\end{array},\right.
$$

where $w_{ \pm}=\left(v_{ \pm}, c_{ \pm}\right)$. Set $w=u+\eta$ and consider the operator

$$
A(u)=D(u+\eta)^{\prime \prime}+c(u+\eta)^{\prime}+F(u+\eta),
$$

acting from $E_{\mu}^{1}$ into $E_{\mu}^{2}$.

Functionalization of the parameter. Solution $w(x)$ of equation (1.2) is invariant with respect to translation in space. Along with any solution $w(x)$, the functions $w(x+h)$ also satisfy this equation for any real $h$. This property of solutions of autonomous problems on the whole axis implies the existence of a zero eigenvalue of the linearized operator $A^{\prime}$. Consequently, we cannot find the index of the solution (the index is understood here as the value of the degree with respect to a small ball containing the solution). Moreover, this family of solutions is not bounded in the weighted norm. Therefore, we cannot apply the Leray-Schauder method to study the existence of solutions.

In order to overcome these difficulties we introduce functionalization of the parameter $c$ [62] (Chapter 2). This means that instead of the unknown constant $c$ we introduce some given functional $c(w)$ such that $c(w(\cdot+h))$ is a monotone function of $h$ with the values from $-\infty$ to $\infty$. Hence, equation $c(w(\cdot+h))=c$ has a unique solution $h$ for any wave speed $c$. Therefore, we obtain an equivalent problem without invariance of solutions with respect to translation in space. The linearized operator $A^{\prime}$ does not have zero eigenvalue.

Homotopy. We consider the operator $A_{\tau}(u)$

$$
A_{\tau}(u)=D(u+\eta)^{\prime \prime}+c(u+\eta)^{\prime}+F_{\tau}(u+\eta),
$$

acting from $E_{\mu}^{1}$ into $E_{\mu}^{2}$ and depending on parameter $\tau \in[0,1]$. We suppose that for $\tau=0$ we have the original operator (2.1) and for $\tau=1$ some model operator for which the degree 
is different from 0 . The function $F_{\tau}(w)$ is bounded and continuous together with its second derivatives with respect to the variables $w$ and $\tau$.

According to the Leray-Schauder (LS) method, we need to obtain a priori estimates of solutions of the equation $A_{\tau}(u)=0$ independent of $\tau$. We will use a modification of the LS for some subclasses of solutions.

Topological degree. The operator linearized about any function in $E_{\mu}^{1}$ satisfies the Fredholm property and has the zero index. The nonlinear operator is proper on closed bounded sets. This means that the inverse image of a compact set is compact in any closed bounded set in $E_{\mu}^{1}$. The topological degree can be defined for this operator. All these properties can be found in $[62,63,64,65,54]$.

\section{Leray-Schauder method on subclasses of solutions}

We consider the operator equation

$$
A_{\tau}(u)=0,
$$

where the operator $A_{\tau}(u): E_{\mu}^{1} \rightarrow E_{\mu}^{2}$ is defined in Section 2. The homotopy is constructed in such a way that $A_{0}(u)$ corresponds to the original problem (1.2), (1.3) and $A_{1}(u)$ to the model problem. In order to apply the Leray-Schauder method, we need to verify two conditions: a priori estimates of solutions of equation (3.1) hold in the space $E_{\mu}^{1}$ and the value of the topological degree for the model operator is different from 0 .

Suppose that the set of solutions $\mathcal{K}$ of equation (3.1) in the space $E_{\mu}^{1}$ can be represented as a union of two subsets $\mathcal{K}_{1}$ and $\mathcal{K}_{2}$ such that the following two conditions are satisfied:

(i) for any $u \in \mathcal{K}_{1}$ and $v \in \mathcal{K}_{2}$, the following estimate holds

$$
\|u-v\|_{E_{\mu}^{1}} \geq r
$$

with some positive constant $r$ independent of $u$ and $v$. We call this property separation of solutions.

(ii) for any $u \in \mathcal{K}_{1}$

$$
\|u\|_{E_{\mu}^{1}} \leq R
$$

with some positive constant $R$ independent of $u$. This is a priori estimate of solutions from the first subset.

Thus, we have a priori estimates of solutions which belong to the class $\mathcal{K}_{1}$. but not of all possible solutions. Therefore, we need to modify the Leray-Schauder method in the following way. Denote by $B$ a ball in the space $E_{\mu}^{1}$ which contains all solutions from the class $\mathcal{K}_{1}$. Since the operator $A_{\tau}(u)$ is proper [54], that is the inverse image of the compact set is compact in 
any bounded closed set, then the set of solutions in $B$ is compact. For each solution $u \in \mathcal{K}_{1}$, consider a ball $b_{r}(u)$ of radius $r$ and center $u$. Set

$$
\Omega_{r}=\cup_{u \in \mathcal{K}} b_{r}(u) .
$$

Let us choose $r$ small enough such that $\Omega_{r}$ contains all solutions from $\mathcal{K}_{1}$ and does not contain other solutions. Consider the topological degree $\gamma\left(A_{\tau}, \Omega_{r}\right)$. It is well defined since $A_{\tau}(u) \neq 0$ for $u \in \partial \Omega_{r}$. We suppose that the degree is different from 0 for the model problem, $\gamma\left(A_{1}, \Omega_{r}\right) \neq 0$. Therefore, $\gamma\left(A_{0}, \Omega_{r}\right) \neq 0$, and equation $A_{0}(u)=0$ has a solution in $\Omega_{r}$.

This approach is applicable if the conditions (i) and (ii) hold. We will present some classes of problems for which these properties are satisfied. Namely, we will illustrate this approach with locally monotone systems [62], [65]-[55] and then we will show other examples.

\subsection{Separation of solutions}

The two subclasses of solutions separated in the function space are monotone and nonmonotone solutions. We will identify some classes of equations for which the properties (i) and (ii) hold.

Definition 3.1. System (1.2) is called locally monotone if for any $i$ and $w$, equality $F_{i}(w)=0$ implies the inequality

$$
\frac{\partial F_{i}}{\partial w_{j}}>0, \quad j=1, \ldots, n, \quad j \neq i .
$$

If this inequality holds for all $w$, then this system is called monotone.

Suppose that (i) is not valid. Then there are two sequences, $u^{i} \in \mathcal{K}_{1}$ (monotone solutions) and $v^{i} \in \mathcal{K}_{2}$ (non-monotone solutions) such that $\left\|u^{i}-v^{i}\right\|_{E_{\mu}^{1}} \rightarrow 0$ as $i \rightarrow \infty$. We will show that this assumption leads to a contradiction.

If condition (ii) is satisfied, then the sequence $u^{i}$ is bounded. From the properness of the operator $A_{\tau}$ on closed bounded sets $[65,54]$ it follows that it has a convergent subsequence. Without loss of generality we can assume that $\left\|u^{i}-w\right\|_{E_{\mu}^{1}} \rightarrow 0$ for some function $w \in E_{\mu}^{1}$. Therefore, $w^{\prime}(x) \leq 0$ for all $x \in \mathbb{R}$ (component-wise). We show that this inequality is strict.

Lemma 3.2. Let $w(x)$ be a solution of a locally monotone system (1.2). If $w^{\prime}(x) \leq 0$ for all $x \in \mathbb{R}$ (component-wise) and $w(x) \not \equiv$ const, then $w^{\prime}(x)<0$.

Proof. Suppose that $w_{i}^{\prime}\left(x_{0}\right)=0$ for some $i=1, \ldots, n$ and $x_{0}$. Then $w_{i}^{\prime \prime}\left(x_{0}\right)=0$. Hence, by virtue of the $i$ th equation of system $(1.2), F_{i}\left(w\left(x_{0}\right)\right)=0$. We set $u_{i}(x)=-w_{i}^{\prime}(x)$ and differentiate the $i$ th equation of system (1.2). Then we get

$$
d_{i} u_{i}^{\prime \prime}+c u_{i}^{\prime}+\frac{\partial F_{i}}{\partial w_{i}} u_{i}-\sum_{j \neq i} \frac{\partial F_{i}}{\partial w_{j}} w_{j}^{\prime}=0 .
$$

Since $\frac{\partial F_{i}}{\partial w_{j}}>0($ see $(3.4))$ and $w_{j}^{\prime}(x) \leq 0$, then 


$$
S\left(x_{0}\right) \equiv-\sum_{j \neq i} \frac{\partial F_{i}}{\partial w_{j}} w_{j}^{\prime}\left(x_{0}\right) \geq 0 .
$$

Assume, first, $w_{i}^{\prime}(x) \not \equiv 0$ in any small interval $I\left(x_{0}\right)$ around $x_{0}$. If we take it sufficiently small, then $S(x) \geq 0$ in $I\left(x_{0}\right)$ by virtue of (3.4) and the inequalities $w_{j}(x)^{\prime} \leq 0$. Hence we obtain a contradiction with the maximum principle for equation (3.5) in $I\left(x_{0}\right)$ since $u_{i}(x) \geq 0$ in $I\left(x_{0}\right), u_{i}\left(x_{0}\right)=0$ and $u_{i}(x) \not \equiv 0$.

If $w_{i}^{\prime}(x) \equiv 0$ in some interval $I_{0}$, then we repeat the previous construction in a slightly larger interval $I$ and obtain a similar contradiction.

Next, we consider the sequence of non-monotone solutions $v^{i}$. For each such solution there is at least one point $x_{i}$ where the derivative of one of the components of the solution vanish. Suppose, first, that this sequence is bounded. From the convergence $\left\|v^{i}-w\right\|_{E_{\mu}^{1}} \rightarrow 0$ as $i \rightarrow \infty$ it follows that the derivative of the limiting function $w^{\prime}(x)$ also vanish (for one of the components). We obtain a contradiction with Lemma 3.2. Therefore, the sequence $x_{i}$ is not bounded. Without loss of generality we can assume that $x_{i} \rightarrow \infty$ as $i \rightarrow \infty$. This gives a contradiction with the following lemma.

Lemma 3.3. Let $v(x)$ be a solution of system (1.2) such that $v(x) \rightarrow 0$ as $x \rightarrow \infty$. Moreover, the matrix $F^{\prime}(0)$ has positive off-diagonal elements and negative principal eigenvalue (i.e. with the maximal real part). If $v^{\prime}\left(x_{0}\right)<0$ (component-wise) for some $x_{0}$ sufficiently large, then $v^{\prime}(x)<0$ for all $x \geq x_{0}$.

Proof. Set $u(x)=-v^{\prime}(x)$ and differentiate equation (1.2):

$$
D u^{\prime \prime}+c u^{\prime}+B(x) u=0,
$$

where $B(x)=F^{\prime}(v(x)), u\left(x_{0}\right)>0, u(x) \rightarrow 0$ as $x \rightarrow \infty$. Since the matrix $F^{\prime}(0)$ has positive off-diagonal elements and negative principal eigenvalue, then $F^{\prime}(0) p<0$, where $p$ is the principal eigenvector. Therefore, we can choose $x_{0}$ sufficiently large such that $B(x) p<0$ for all $x \geq x_{0}$.

We need to prove that $u(x)>0$ for $x \geq x_{0}$. Suppose that this is not true. If $u(x) \geq 0$ for all $x \geq x_{0}$ and $u_{j}\left(x_{1}\right)=0$ for some $j$ and $x_{1}$, then we obtain a contradiction with the maximum principle. Therefore, we consider the case where one of the components of the function $u(x)$ becomes negative. Hence there exists a positive number $t$ such that the function $\hat{u}(x)=u(x)+t p$ satisfies the following conditions: $\hat{u} \geq 0$ for all $x \geq x_{0}, \hat{u}\left(x_{0}\right)>0$, $\hat{u}_{j}\left(x_{2}\right)=0$ for some $j$ and $x_{2}>x_{0}$. It satisfies the following equation:

$$
D \hat{u}^{\prime \prime}+c \hat{u}^{\prime}+B(x) \hat{u}+f(x)=0,
$$

where $f(x)=-t B(x) p>0$. Therefore, we obtain again a contradiction with the maximum principle. This contradiction proves the lemma. 
Thus, we have proved the following theorem.

Theorem 3.4. Let the system

$$
D w^{\prime \prime}+c w^{\prime}+F_{\tau}(w)=0
$$

be locally monotone, $F_{\tau}\left(w_{ \pm}\right)=0$ for some $w_{ \pm}, w_{+}<w_{-}$(component-wise), and the matrices $F_{\tau}^{\prime}\left(w_{ \pm}\right)$have all eigenvalues in the left-half plane. Suppose that for any monotonically decreasing solution $w_{m}$ of system (3.8) with the limits

$$
w( \pm \infty)=w_{ \pm}
$$

the estimate

$$
\left\|w_{m}-\eta\right\|_{E_{\mu}^{1}} \leq R
$$

holds with some positive constant $R$ independent of the solution and of the value of $\tau \in[0,1]$. Then there exists a positive constant $r$ such that

$$
\left\|w_{m}-w_{n}\right\|_{E_{\mu}^{1}} \geq r
$$

where $w_{n}$ is any non-monotone solution of problem (3.8), (3.9) possibly for a different value of $\tau, r$ does not depend on solutions and on $\tau$.

Remarks 3.5. From the condition of local monotonicity it follows that the matrices $F^{\prime}\left(w_{ \pm}\right)$ have positive off-diagonal elements. Therefore, Perron-Frobenius theorem affirms that their principal eigenvalues are real and simple, the corresponding eigenvectors are positive. These properties were used in Lemma 3.3.

It is important to note that monotone systems satisfy the maximum principle but locally monotone systems do not satisfy it. However, the property of separation of solutions remains valid for them. Inequality (3.4) in the definition of local monotonicity can be non-strict.

Let us also recall that the wave speed in (3.8), (3.9) can depend on parameter $\tau, c=c_{\tau}$.

\subsection{Estimates of solutions}

In this section we will obtain a priori estimates of monotone solutions in weighted Hölder spaces. Since the principal eigenvalues of the matrices $F_{\tau}^{\prime}\left(w_{ \pm}\right)$are negative, then the solutions converge to their limiting values at infinity exponentially. In the other words, the following estimates hold:

$$
\left|w_{m}(x)-\eta(x)\right| \leq K_{1} e^{-\mu_{0} x}, \quad x \geq N_{+}, \quad\left|w_{m}(x)-\eta(x)\right| \leq K_{1} e^{\mu_{0} x}, \quad x \leq N_{-}
$$

with some positive constants $K_{1}$ and $\mu_{0}$ independent of a monotone solution $w_{m}$ and the value of $\tau$. On the contrary, the values $N_{+}$and $N_{-}$can depend on the solution. They are chosen in such a way that 


$$
\left|w_{m}(x)-\eta(x)\right| \leq \epsilon, \quad x \geq N_{+}, \quad\left|w_{m}(x)-\eta(x)\right| \leq \epsilon, \quad x \leq N_{-}
$$

for some small positive $\epsilon$. This means that estimates (3.12) hold in some neighborhoods of the points $w_{ \pm}$in $\mathbb{R}^{n}$ (w-space). This property follows from the classical results on behavior of solutions of the corresponding first-order ordinary differential system of equations in the vicinity of stationary points.

Since the weight function $\mu(x)$ has polynomial growth at infinity, then we obtain the estimate

$$
\left|\left(w_{m}(x)-\eta(x)\right) \mu(x)\right| \leq K_{2}
$$

for $x \geq N_{+}$and $x \leq N_{-}$. If $N_{+}$and $N_{-}$are uniformly bounded for all solutions, then the last estimate obviously holds for all $x \in \mathbb{R}$.

Let us consider the case where these values are not uniformly bounded. Suppose that $N_{+}^{i} \rightarrow \infty$ for some sequence of solutions $w^{i}$, and $N_{-}^{i}$ remains bounded. Consider the shifted functions $v^{i}(x)=w^{i}\left(x-N_{+}^{i}\right)$. We have the equality $\left|v^{i}(0)-\nu(0)\right|=\epsilon$. We can choose a subsequence of the sequence $v^{i}(x)$ locally converging to some limiting function $v^{0}(x)$. It is a solution of system (3.8) for some $\tau$, it is monotonically decreasing, and $\left|v^{0}(0)-\nu(0)\right|=\epsilon$. Hence $v^{0}(x) \rightarrow w_{+}$as $x \rightarrow \infty$, and there exists a limit $v^{*}=v^{0}(-\infty)$. Clearly, $F\left(v^{*}\right)=0$. Since $N_{+}^{i}-N_{i}^{i} \rightarrow \infty$, then $\left|v^{*}-w_{-}\right| \geq \epsilon$. Thus, we have constructed a solutions with the limits

$$
v^{0}(-\infty)=v^{*}, \quad v^{0}(\infty)=w_{+}, \quad v^{*} \neq w_{ \pm} .
$$

Similarly, for the shifted functions $u^{i}(x)=w^{i}\left(x-N_{-}^{i}\right)$ we obtain a limiting solution $u^{0}(x)$ with the limits

$$
u^{0}(-\infty)=w_{-}, \quad u^{0}(\infty)=v_{*}, \quad v_{*} \neq w_{ \pm} .
$$

We can now prove the following theorem.

Theorem 3.6. Let the system (3.8) be locally monotone, $F_{\tau}\left(w_{ \pm}\right)=0$ for some $w_{ \pm}, w_{+}<$ $w_{-}$, and the matrices $F_{\tau}^{\prime}\left(w_{ \pm}\right)$have all eigenvalues in the left-half plane. Suppose that for any other zero $w^{0}$ of the function $F(w)$ such that $w_{+} \leq w^{0} \leq w_{-}$, the principal eigenvalue of the matrix $F^{\prime}\left(w^{0}\right)$ is positive. Then the estimate

$$
\sup _{x}\left|\left(w_{m}(x)-\eta(x)\right) \mu(x)\right| \leq K
$$

holds for any monotonically decreasing solution $w_{m}(x)$ of problem (3.8), (3.9) with a constant $K$ independent of solution.

Proof. Suppose that the assertion of the theorem does not hold. Then, as it is shown above, the values $N_{ \pm}$in (3.12) are not uniformly bounded. Suppose that there is a sequence of solutions $w^{i}$ for which $N_{+}^{i} \rightarrow \infty$ as $i \rightarrow \infty$, and $N_{-}^{i}$ remain bounded. Then there are 
solutions $v^{0}(x)$ with limits $(3.14)$ and $u^{0}(x)$ with limits (3.15). The existence of the former implies that $c<0$ and of the latter that $c>0$ ([58,62], Lemma 2.8, Chapter 3, p. 165). This contradiction proves that the assumption on $N_{ \pm}$cannot hold.

Similarly, we can consider the case where $N_{-}^{i}$ tends to $-\infty$ and $N_{+}^{i}$ remains bounded, or both of them are unbounded. Since the solutions are invariant with respect to translation in space, all these cases can be reduced to the case where the values $N_{-}^{i}$ are bounded. The shift remains bounded due to a priori estimates of the wave speed [58, 62].

Corollary 3.7. Let $u=w_{m}-\eta$, where $w_{m}$ is a monotone solution of problem (3.8), (3.9). Then $\|u\|_{E_{\mu}^{1}} \leq K$, where a positive constant $K$ does no depend on the solution.

Thus, we obtain a priori estimates of monotone solutions.

\section{Existence of pulses and waves}

In this section we will review the results on the existence of solutions obtained by the method presented above. We begin with the scalar equation for which the existence of solutions can be studied by elementary methods and which allows us to explain the interconnection between the existence of wave and pulses. Some of these results can be generalized by the method of monotone solutions for the systems of equations.

\subsection{Pulses and waves for the scalar equation}

Consider the problem

$$
w^{\prime \prime}+c w^{\prime}+F(w)=0, \quad w( \pm \infty)=w_{ \pm}
$$

where $w(x)$ is the scalar function, $c$ is a constant, the wave speed, the function $F(w)$ is bounded and continuous together with its second derivatives, $F\left(w_{ \pm}\right)=0, F^{\prime}\left(w_{ \pm}\right)<0$. Solutions of this problem are called travelling waves.

Theorem 4.1. Suppose that $F(w)<0$ for $w_{+}<w<w_{0}$ and $F(w)>0$ for $w_{0}<w<$ $w_{-}$. Then problem (4.1) has a solution $w(x)$ for a unique value of $c$. It is monotonically decreasing, and $c \gtreqless 0$ if and only if $\int_{w_{+}}^{w_{-}} F(w) d w \gtreqless 0$.

The proof of the existence of solutions follows from the elementary phase plane analysis of the first-order system of equations

$$
w^{\prime}=p, \quad p^{\prime}=-c p-F(w)
$$

equivalent to equation (4.1) (see, e.g., [62]). The sign of the wave speed can be determined if we multiply equation (4.1) and integrate over the whole axis.

Next, consider the problem 


$$
w^{\prime \prime}+F(w)=0, \quad w( \pm \infty)=w_{+},
$$

similar to (4.1) with $c=0$ and with the equal limits at infinity. Nontrivial solutions of this problem are called pulses.

Theorem 4.2. Suppose that $F(w)<0$ for $w_{+}<w<w_{0}$ and $F(w)>0$ for $w_{0}<w<w_{-}$. Then problem (4.2) has a pulse solution $w(x)>w_{+}$if and only if $\int_{w_{+}}^{w_{-}} F(w) d w>0$.

The proof of this theorem can be done by an explicit construction of solution of the corresponding first-order system.

Corollary 4.3. The pulse, that is the solution of problem (4.2) with $w>w_{+}$, exists if and only if the wave, that is the solution of problem (4.1), has a positive speed.

\subsection{Monotone and locally monotone systems}

The existence results formulated above for the scalar equation can be generalized (with some restrictions) for locally monotone systems.

Theorem 4.4. Let system (1.2) be locally monotone, $F\left(w_{ \pm}\right)=0$ for some $w_{ \pm}, w_{+}<w_{-}$, and the matrices $F^{\prime}\left(w_{ \pm}\right)$have all eigenvalues in the left-half plane. Suppose that for any other zero $w^{0}$ of the function $F(w)$ such that $w_{+} \leq w^{0} \leq w_{-}$, the principal eigenvalue of the matrix $F^{\prime}\left(w^{0}\right)$ is positive. Then there exists a monotonically decreasing solution of problem (1.2), (1.3) for some values of $c$. If the system is monotone, then such value of $c$ is unique.

The proof of this theorem is based on the method of monotone solutions presented above. The model system and the homotopy can be found in [58, 62]. Theorem 4.2 on the existence of pulses is generalized for monotone systems of two equations [35, 36]:

Theorem 4.5. Let the system

$$
D w^{\prime \prime}+F(w)=0,
$$

where $w=\left(w_{1}, w_{2}\right), F=\left(F_{1}, F_{2}\right)$, be monotone, $F\left(w_{ \pm}\right)=0$, and the principal eigenvalues of the matrices $F^{\prime}\left(w_{ \pm}\right)$be negative. There is a single zero $w^{0}$ of the function $F$ such that $w_{+} \leq w^{0} \leq w_{-}$, and the principal eigenvalues of the matrices $F^{\prime}\left(w_{ \pm}\right)$is positive. Moreover, suppose that $F_{1}(w)=0\left(F_{2}(w)=0\right)$ if and only if $w_{1}=f_{1}\left(w_{2}\right)\left(w_{2}=f_{2}\left(w_{1}\right)\right)$, where $f_{i}^{\prime}(s)>0, i=1,2$. Then there exists a solution $w(x)$ of system (4.3) with the limits $w( \pm \infty)=w_{+}, w(x)>w_{+}$for $x \in \mathbb{R}$ if and only if the wave speed (Theorem 4.4) is positive.

Thus, the existence of pulses is proved only for the system of two equations under some additional conditions. The difficulty in proving a similar result for general monotone systems, and also for locally monotone systems, consists in the choice of model problem and in the construction of the homotopy with a priori estimates of solutions. Existence of pulses is also proved for a non-autonomous scalar equation [22] and for the system of equations describing blood coagulation [31]. 
The system of competition of species. The system of competition of species is a system of two equations where

$$
F_{1}\left(w_{1}, w_{2}\right)=w_{1}\left(1-w_{1}-a w_{2}\right), \quad F_{2}\left(w_{1}, w_{2}\right)=w_{2}\left(1-b w_{1}-w_{2}\right) .
$$

It provides a good example for the illustration of the existence of waves and pulses. The function $F(w)$ has up to four non-negative zeros, $P_{0}=(0,0), P_{1}=(1,0), P_{2}=(0,1), P_{3}=$ $\left(w_{1}^{0}, w_{2}^{0}\right)$, where the values $w_{1}^{0}, w_{2}^{0}$ are determined as a solution of the system of equations $w_{1}+a w_{2}=1, b w_{1}+w_{2}=1$. The point $P_{0}$ is unstable, the points $P_{1}$ and $P_{2}$ are stable if $a>1, b>1$. In this case the point $P_{3}$ is unstable. We set $w_{+}=P_{1}, w_{-}=P_{2}$.

The off-diagonal elements of the matrix $F^{\prime}(w)$ are negative. The system can be reduced to the monotone system by the change of variables $u_{1}=w_{1}, u_{2}=1-w_{2}$. Existence of waves and pulses follows from Theorems 4.4, 4.5 with some additional technical details which can be found in $[58,62]$.

If we consider a more general nonlinearity

$$
F_{1}\left(w_{1}, w_{2}\right)=w_{1} \phi_{1}\left(w_{1}, w_{2}\right)\left(1-w_{1}-a w_{2}\right), \quad F_{2}\left(w_{1}, w_{2}\right)=w_{2} \phi_{2}\left(w_{1}, w_{2}\right)\left(1-b w_{1}-w_{2}\right)
$$

with some positive and sufficiently smooth functions $\phi_{i}\left(w_{1}, w_{2}\right), i=1,2$, then this system remains locally monotone after the change of variables, but it is not a monotone system. We still have the existence of waves from Theorem 4.4 but Theorem 4.5 is not applicable, and the existence of pulses is not proved.

A similar system of three or more equations can be reduced to a monotone system under some additional conditions. Consider, as example, the system of three equations with the nonlinearity

$$
\begin{gathered}
F_{1}(w)=w_{1}\left(1-w_{1}-a_{2} w_{2}+a_{3} w_{3}\right), \quad F_{2}(w)=w_{2}\left(1-b_{1} w_{1}-w_{2}-b_{3} w_{3}\right) \\
F_{3}(w)=w_{3}\left(1+c_{1} w_{1}-c_{2} w_{2}-w_{3}\right),
\end{gathered}
$$

where $w=\left(w_{1}, w_{2}, w_{3}\right)$, and $a_{i}, b_{i}, c_{i}$ are some positive constants. After the change of variables $u_{1}=w_{1}, u_{2}=1-w_{2}, u_{3}=w_{3}$ we get the system

$$
D u^{\prime \prime}+c u^{\prime}+G(u)=0
$$

where

$$
\begin{gathered}
G_{1}(u)=u_{1}\left(1-u_{1}-a_{2}\left(1-u_{2}\right)+a_{3} u_{3}\right), \quad G_{2}(u)=\left(1-u_{2}\right)\left(b_{1} u_{1}-u_{2}+b_{3} u_{3}\right), \\
G_{3}(u)=u_{3}\left(1+c_{1} u_{1}-c_{2}\left(1-u_{2}\right)-u_{3}\right) .
\end{gathered}
$$

This system satisfies the monotonicity condition for $u_{1}, u_{3}>0, u_{2}<1$. We consider the points $w_{+}=(0,0,0)$ and $w_{-}=\left(w_{1}^{0}, w_{2}^{0}, w_{3}^{0}\right)$, where $w_{1}^{0}, w_{2}^{0}, w_{3}^{0}$ is a solution of the linear algebraic system of equations $A u=q$, where 


$$
A=\left(\begin{array}{ccc}
-1 & a_{2} & a_{3} \\
b_{1} & -1 & b_{3} \\
c_{1} & c_{2} & -1
\end{array}\right), \quad q=\left(\begin{array}{c}
1-a_{2} \\
0 \\
1-c_{2}
\end{array}\right)
$$

It can be verified that the point $w_{+}$is stable if $a_{2}>1, c_{2}>1$. If the principal eigenvalue of the matrix $A$ is negative, then $w_{-}$is positive and also stable. In this case, existence of waves follows from Theorem 4.4. However, we cannot state the existence of pulses since Theorem 4.5 is applicable only for two equations. The form of nonlinearity (4.4) implies that the first and the third species are in cooperation, and each of them is in competition with the second species.

\subsection{Multi-dimensional equations and systems}

If we consider wave propagation in unbounded cylinders, then instead of equation (1.2) we have the equation

$$
D \Delta w+c \frac{\partial w}{\partial x_{1}}+F\left(w, x^{\prime}\right)=0,
$$

where $x_{1}$ is the variable along the axis of the cylinder, $x^{\prime}$ is the variable in the cross-section. This equation is completed by Dirichlet or Neumann conditions at the boundary of the cylinder. The problem in the cross-section of the cylinder,

$$
D \Delta^{\prime} w+F\left(w, x^{\prime}\right)=0
$$

is supposed to have two solutions $w_{ \pm}\left(x^{\prime}\right)$ for which the corresponding linearized operator has all eigenvalues in the left-half plane. Then we have the bistable case and we look for a solution of equation (4.6) with the limits

$$
\lim _{x_{1} \rightarrow \pm \infty} w(x)=w_{ \pm}\left(x^{\prime}\right)
$$

In the multi-dimensional case separation of solutions can be done for the monotone systems but not for the locally monotone systems. Therefore, existence of waves is proved for the scalar equations and for the monotone systems [59]. The problems with nonlinear boundary conditions arising in some biomedical applications are studied in $[5,8,9]$.

\subsection{Nonlocal and delay equations}

In this section we present the existence results for nonlocal and delay reaction-diffusion equations obtained by the method of monotone solutions. It is similar to the approach described above for the locally monotone systems though some technical details can be different. 


\subsubsection{Nonlocal equations}

In order to introduce nonlocal equations, let us consider the scalar equation (1.1) with a particular form of the nonlinearity

$$
F(u)=u^{2}(1-a u)-\sigma u .
$$

In population dynamics, the first term in the right-hand side corresponds to sexual reproduction of the population and the second term to its mortality. The function $F(u)$ can have from one to three non-negative zeros. In the latter case, the results of Section 4.1 are applicable to it. The reproduction term is proportional to available resources $(1-a u)$, where the linear term describes consumption of resources. In the case of nonlocal or global consumption of resources, instead of (4.9) we have

$$
F(u, I(u))=u^{2}(1-a I(u))-\sigma u
$$

where

$$
I(u)=\int_{-\infty}^{\infty} \phi(x-u) u(y, t) d y
$$

$\phi(x)$ has a finite support (nonlocal consumption) or $\phi(x) \equiv 1$ (global consumption) [14]. Consider, as example, $\phi(x)=\psi(x) /(2 N)$, where

$$
\psi(x)=\left\{\begin{array}{ll}
1 & , \quad|x| \leq N \\
0 & , \quad|x|>N
\end{array} .\right.
$$

In the limit of small $N$, nonlocal consumption (4.10) is formally reduced to the local consumption (4.9). Existence of waves and pulses can be proved for small $N$ by perturbation methods [69]. If $\phi(x) \equiv \psi(x)$, then in the limit of large $N(\phi(x) \equiv 1)$, the waves do not exist, and the existence of pulses can be easily verified analytically. This allows us to prove their existence for sufficiently large $N$. Transition between waves for small $N$ and pulses for large $N$ occurs through the periodic waves and nonlocal bifurcations [56].

System with global consumption. The system of two equations with global consumption in the stationary case writes

$$
d_{1} u^{\prime \prime}+u v(1-a I(u)-b I(v))=0, \quad d_{2} v^{\prime \prime}+u v(1-c I(u)-d I(v))=0 .
$$

The existence of pulses, that is positive solutions of this system with zero limits at infinity is proved by the method of monotone solutions [57]. If the coefficients in the two equations are equal to each other, then system (4.11) can be reduced to the single equation (cf. (4.10)) where the existence of pulses is obvious. If the coefficients are different, the proof of the existence becomes much more involved and requires some sophisticated a priori estimates. 
Scalar equation with nonlocal reproduction. Let us now consider another generalization of (4.9),

$$
F(u, I(u))=u I(u)(1-a u)-\sigma u
$$

where the kernel of the integrala $I(u)$ is a non-negative function with a finite support. In this case the method of monotone solutions is applicable, and the existence of waves is proved in $[7,19]$.

\subsubsection{Delay equation}

Delay reaction-diffusion equation

$$
\frac{\partial u}{\partial t}=D \frac{\partial^{2} u}{\partial x^{2}}+F\left(u, u_{\tau}\right)
$$

where $u_{\tau}(x, t)=u(x, t-\tau)$,

$$
F\left(u, u_{\tau}\right)=u(1-u)-f\left(u_{\tau}\right) u
$$

describes the propagation of viral infection in the tissue [15]. The first term in the nonlinearity describes its reproduction and the second one its mortality due to the immune response. The density of immune cells $f\left(u_{\tau}\right)$ is determined by virus concentration at time $t-\tau$.

The travelling wave solution, $u(x, t)=w(x-c t)$, satisfies the second-order delay equation

$$
D w^{\prime \prime}+c w^{\prime}+w(1-w-f(w(x+c \tau)))=0 .
$$

The unknown constant $c$, the wave speed, enters here the nonlinearity due to time delay in the equation. If $f(u)$ is a monotonically decreasing function, then the maximum principle is applicable to this equation, and it can be used in the proof of wave existence. However, if this function is not decreasing, and this is the case of the model of immune response, then this approach cannot be used. The existence of waves is proved by the method of monotone solutions [49].

\section{Discussion}

Bistability and essential spectrum. The construction of the topological degree implies that the essential spectrum lies in the left-half plane of the complex plane. Therefore, we need to assume that the matrices $F^{\prime}\left(w_{ \pm}\right)$have all eigenvalues with negative real parts. This is the so-called bistable case. In the monostable case, one of them has eigenvalues with a positive real part. The essential spectrum is partially in the right-half plane, and the same degree construction cannot be employed. The degree can be defined in the monostable case by the introduction of an exponential weight that moves the essential spectrum to the left-half plane. This approach was not used to investigate the existence of waves. 
Let us also note that in the bistable case the wave is unique or they form a discrete set of solutions. In the monostable case, there are continuous families of solutions. This is related to the index of the corresponding operators.

Other approaches to prove the existence of waves. The method presented here uses the topological degree for elliptic operators in unbounded domains. It implies the degree construction and a priori estimates of solutions in appropriate weighted spaces. Another approach to prove the existence of travelling waves for the scalar reaction-diffusion equation is developed in $[12,13]$. In order to prove the existence of solutions in an unbounded cylinder, first, it is proved for a bounded part of the cylinder using the LS degree. Uniform estimates of solutions allow the passage to the limit as this bounded part of the cylinder tends to infinity.

Systems of waves. The results on the existence of waves presented above are obtained under the assumption that all zeros of the vector-function $F(w)$ in the rectangle $w_{+} \leq w \leq$ $w_{-}$are unstable, except for $w_{+}$and $w_{-}$. Suppose now that there is a stable zero $w^{0}, w_{+}<$ $w^{0}<w_{-}$. Then, according to Theorem 4.4 , there exists a $\left[w_{+}, w^{0}\right]$-wave and a $\left[w^{0}, w_{-}\right]$-wave, that is the waves with the limits $w(\infty)=w_{+}, w(-\infty)=w^{0}$ and $w(\infty)=w^{0}, w(-\infty)=w_{-}$, respectively. Denote their speeds by $c_{+}$and $c_{-}$. Then the $\left[w_{+}, w_{-}\right]$-wave exists if and only if $c_{-}>c_{+}$. This result is obtained for the scalar equation $[24,25,52,53]$ and for monotone systems [55]. If $c_{-} \leq c_{+}$, then this common wave does not exist, and there are two waves propagating one after another with different speeds.

Limitations and further developments. The method of monotone solutions is based on two properties: separation of monotone and non-monotone solutions and on a priori estimates of monotone solutions. These properties can be proved for some particular classes of problems, and they do not hold in general. Other methods to prove the existence of waves and pulses are also developed only for some particular models. The method of monotone solutions is applicable in particular for monotone and locally monotone systems which have numerous applications. Some of them are presented in this work, some other can be found in $[55,62]$. There are some recent applications for the delay equation without the maximum principle [49].

\section{Acknowledgements}

The first author was partially supported by the Ministry of Education and Science of the Russian Federation (the agreement number 02.a03.21.0008) and by the program PICS CNRS 6583 Matbio. 


\section{References}

[1] S. Agmon, A. Douglis, L. Nirenberg. Estimates near the boundary for solutions of elliptic partial diffrential equations satisfying general boundary conditions. Comm. Pure Appl. Math., 12 (1959), 623-727.

[2] S. Agmon, A. Douglis, L. Nirenberg. Estimates near the boundary for solutions of elliptic partial differential equations satisfying general boundary conditions II. Comm. Pure Appl. Math., 17 (1964), 35-92.

[3] N. Apreutesei, A. Ducrot, V. Volpert. Travelling waves for integro-differential equations in population dynamics. DCDS B, 11 (2009), No. 3, 541-561.

[4] A. Apreutesei, A. Ducrot, V. Volpert. Competition of species with intra-specific competition. Math. Model. Nat. Phenom., 3 (2008), No. 4, 1-27.

[5] N. Apreutesei, A. Tosenberger, V. Volpert. Existence of Reaction-Diffusion Waves with Nonlinear Boundary Conditions. Math. Model. Nat. Phenom., 8 (2013), no. 4, 2-17.

[6] N. Apreutesei, V. Volpert. Properness and topological degree for nonlocal reaction-diffusion operators. Abstract and Applied Analysis, 2011, 1-21.

[7] N. Apreutesei, V. Volpert. Existence of travelling waves for a class of integro-differential equations from population dynamics. International Electronic Journal of Pure and Applied Mathematics, Volume 5 No. 2 2012, 53-67.

[8] N. Apreutesei, V. Volpert. Reaction-diffusion waves with nonlinear boundary conditions. Nonlinear and heterogeneous medium, 8 (2013), no. 1, 23-35.

[9] N. Apreutesei, V. Volpert. Travelling waves for reaction-diffusion problems with nonlinear boundary conditions. Application to a model of atherosclerosis. PAFA, 2017, in press.

[10] P. Benevieri, M. Furi. A simple notion of orientability for Fredholm maps of index zero between Banach manifolds and degree theory. Annales des Sciences Mathématiques de Québec, 22 (1998), 131-148.

[11] P. Benevieri, M. Furi. On the concept of orientability for Fredholm maps between real Banach manifolds. Topol. Methods Nonlinear Anal., 16 (2000), no. 2, 279-306.

[12] H. Berestycki, B. Larrouturou, P. L. Lions. Multidimensional traveling wave solutions of a flame propagation model. Arch. Rational Mech. Anal., 111 (1990), 97-117.

[13] H. Berestycki, L. Nirenberg. Travelling fronts in cylinders. Annales de l'IHP. Analyse non linéaire, 9 (1992), no. 5, 497-572.

[14] N. Bessonov, N. Reinberg, V. Volpert. Mathematics of Darwins Diagram. Math. Model. Nat. Phenom., Vol. 9, No. 3, 2014, 5-25.

[15] G. Bocharov, A. Meyerhans, N. Bessonov, S. Trofimchuk, V. Volpert. Spatiotemporal dynamics of virus infection spreading in tissues. PlosOne, DOI:10.1371/journal.pone.0168576 December $20,2016$. 
[16] J. Borisovich, V. Zvyagin, J. Sapronov. Nonlinear Fredholm maps and the Leray-Schauder theory. Uspehi Mat. Nauk, 32 (1977), 3-54; English translation in Russian Math. Surveys, 32 (1977), 1-54.

[17] J.F. Collet, V. Volpert. Computation of the index of linear elliptic operators in unbounded cylinders. J. Funct. Analysis, 1999, 164, pp. 34-59.

[18] E.N. Dancer. Boundary value problems for ordinary differential equations on infinite intervals. Proc. London Math. Soc., 30 (1975), No. 3, 76-94.

[19] I. Demin, V. Volpert. Existence of Waves for a Nonlocal Reaction-Diffusion Equation. Math. Model. Nat. Phenom., 5 (2010), No. 5, 80-101.

[20] K. D. Elworthy, A. J. Tromba. Degree theory on Banach manifolds. In: Nonlinear Functional Analysis, F.E. Browder, Ed., Proc. Symp. Pure Math., Vol. 18, Part I, 1970, 86-94.

[21] K. D. Elworthy, A.J. Tromba. Differential structures and Fredholm maps on Banach manifolds. In: Global Analysis, S.S. Chern, S. Smale, Eds., Proc. Symp. Pure Math., 15 (1970), 45-94.

[22] N. Eymard, V. Volpert, V. Vougalter. Existence of pulses for local and nonlocal reactiondiffusion equations. Journal of Dynamics and Differential Equations, 29 (2017), no. 3, 11451158 .

[23] C. Fenske. Analytische Theorie des Abbildungrades fur Abbildungen in Banachraumen. Math. Nachr., 48 (1971), 279-290.

[24] P. C. Fife, J. B. McLeod. The approach to solutions of nonlinear diffusion equations to traveling front solutions. Arch. Ration. Mech. Anal., 65 (1977), 335-361.

[25] P. C. Fife, J. B. McLeod. A phase plane discussion of convergence to travelling fronts for nonlinear diffusion. Arch. Rational Mech. Anal., 75 (1981), 281-314.

[26] P.M. Fitzpatrick. The parity as an invariant for detecting bifurcaton of the zeroes of one parameter families of nonlinear Fredholm maps. In: Topological Methods for Ordinary Differential Equations, M. Furi, P. Zecca, Eds., Lecture Notes in Math., Vol. 1537, 1993, 1-31.

[27] P.M. Fitzpatrick, J. Pejsachowicz. Parity and generalized multiplicity. Trans. Amer. Math. Soc., 326 (1991), 281-305.

[28] P.M. Fitzpatrick, J. Pejsachowicz. Orientation and the Leray-Schauder degree for fully nonlinear elliptic boundary value problems. Mem. Amer. Math. Soc., 483 (1993).

[29] P.M. Fitzpatrick, J. Pejsachowicz, P.J. Rabier. The degree of proper $C^{2}$ Fredholm mappings. J. Reine Angew., 427 (1992), 1-33.

[30] P.M. Fitzpatrick, J. Pejsachowicz, P.J. Rabier. Orientability of Fredholm families and topological degree for orientable nonlinear Fredholm mappings. J. Funct. analysis, 124 (1994), $1-39$.

[31] T. Galochkina, M. Marion, V. Volpert. Initiation of reaction-diffusion waves of blood coagulation. Submitted. 
[32] R.A. Gardner. Existence and stability of traveling wave solution of competition models: a degree theoretic approach. J. Differential Equations 44 (1982), 343-364.

[33] C.A. Isnard. Orientation and degree in infinite dimensions. Notices Amer. Math. Soc., 19 (1972), A-514.

[34] J. Leray, J. Schauder. Topologie et équations fonctionnelles. Annales scientifiques de l'Ecole Normale Supérieure Sér. 3, 51 (1934), 45-78.

[35] M. Marion, V. Volpert. Existence of pulses for a monotone reaction-diffusion system Pure and Applied Functional Analysis, 1 (2016), no. 1, 97-122.

[36] M. Marion. V. Volpert. Existence of Pulses for the System of Competition of Species. JDDE, 2017 , in press.

[37] C. Miranda. Equazioni alle derivate parziale di tipo elliptico. Springer-Verlag, Berlin, 1955.

[38] A. Muravnik. On the half-plane Dirichlet problem for differential-difference elliptic equations with several nonlocal terms. Math. Model. Nat. Phenom., 12 (2017), no. 6.

[39] G.G.Onanov, A.L.Skubachevskii. Nonlocal problems in the mechanics of three-layer shell.s Math. Model. Nat. Phenom., 12 (2017), no. 6.

[40] V. Rabinovich. Pseudodifferential operators in unbounded domains with conical structure at infinity. Mat. Sb. (N.S.), 80 (122) (1969), 77-96.

[41] V.S. Rabinovich. The Fredholm property of general boundary value problems on noncompact manifolds, and limit operators. Dokl. Akad. Nauk, 325 (1992), No. 2, 237-241. English translation: Russian Acad. Sci. Dokl. Math., 46 (1993), No. 1, 53-58.

[42] P.J. Rabier, C.A. Stuart. Fredholm and properness properties of quasilinear elliptic operators on $R^{N}$. Math. Nachr., 231 (2001), 129-168.

[43] V. Rabinovich, S. Roch, B. Silbermann. Limit operators and their applications in operator theory. Operator Theory: Advances and Applications, 150. Birkhauser Verlag, Basel, 2004.

[44] L. Rossovskii Elliptic functional differential equations with incommensurable contractions. Math. Model. Nat. Phenom., 12 (2017), no. 6.

[45] A.L. Skubachevskii. Nonlocal elliptic problems and multidimensional diffusion processes. Russian J. Math. Phys., 3 (1995), no. 3, 327-360.

[46] A.L. Skubachevskii Elliptic Functional Differential Equationa and Its Applications. Birkhauser, Basel, 1997.

[47] S. Smale. An infinite dimensional version of Sard's theorem. Amer. J. Math., 87 (1965), 861866.

[48] A. Tasevich. Analysis of functional-differential equation with orthotropic contractions. Math. Model. Nat. Phenom., 12 (2017), no. 6. 
[49] S. Trofimchuk, V. Volpert. Travelling waves for a bistable reaction-diffusion equation with delay. http://arxiv.org/abs/1701.08560v1

[50] M.I. Vishik. On strongly elliptic systems of differential equations. Mat. Sb., 29 (1951), 615-676.

[51] L.R. Volevich. Solvability of boundary problems for general elliptic systems. Mat. Sbor., 68 (1965), 373-416; English translation: Amer. Math. Soc. Transl., 67 (1968), Ser. 2, 182-225.

[52] V. Volpert. Asymptotic behavior of solutions of a nonlinear diffusion equation with a source term of general form. Siberian Math. J., 30 (1989) no. 1, 25-36.

[53] V. Volpert. Convergence to a wave of solutions of a nonlinear diffusion equation with source of general type. Siberian Math. J., 30 (1989) no.2, 203-210.

[54] V. Volpert. Elliptic partial differential equations. Volume 1. Fredholm theory of elliptic problems in unbounded domains. Birkhäuser, Basel, 2011.

[55] V. Volpert. Elliptic partial differential equations. Volume 2. Reaction-diffusion equations. Birkhäuser, Basel, 2014.

[56] V. Volpert. Pulses and waves for a bistable nonlocal reaction-diffusion equation. Applied Mathematics Letters, 44 (2015), 21-25.

[57] V. Volpert, N. Reinberg, M. Benmir, S. Boujena. On pulse solutions of a reactiondiffusion system in population dynamics Nonlinear Analysis, 120 (2015), 76-85.

[58] A.I. Volpert, V.A. Volpert. Applications of the rotation theory of vector fields to the study of wave solutions of parabolic equations. Trans. Moscow Math. Soc., 1990, 52, pp. 59-108.

[59] A. Volpert, V. Volpert. Existence of multidimensional travelling waves and systems of waves. Comm. PDE, 2001, Volume 26, Numbers 3-4, pp. 421 - 459.

[60] A. Volpert, V. Volpert. Formally adjoint problems and solvability conditions for elliptic operators. Russian Journal of Mathematical Physics, Vol. 11, No. 4, 2004, pp. 474-497.

[61] A. Volpert, V. Volpert. Fredholm property of elliptic operators in unbounded domains. Trans. Moscow Math. Soc. (2006) 67, 127-197.

[62] A. Volpert, Vit. Volpert, Vl. Volpert. Traveling wave solutions of parabolic systems. Translation of Mathematical Monographs, Vol. 140, Amer. Math. Society, Providence, 1994.

[63] A.I. Volpert, V.A. Volpert. The construction of the Leray-Schauder degree for elliptic operators in unbounded domains. Annales de l'IHP. Analyse non lineaire, 1994,11, No. 3, pp. 245-273.

[64] V. Volpert, A. Volpert, J.F. Collet. Topological degree for elliptic operators in unbounded cylinders. Adv. Diff. Eq., 1999, 4, No. 6, 777-812.

[65] A. Volpert, V. Volpert. Properness and topological degree for general elliptic operators. Abstract and Applied Analysis, 2003, no. 3, 129-181. 
[66] V. Vougalter, V. Volpert. Solvability conditions for some non-Fredholm operators. Proc. Edinb. Math. Soc., 54 (2011), no. 1, 249-271.

[67] V. Vougalter, V. Volpert. Solvability relations for some non Fredholm operators. Int. Electron. J. Pure Appl. Math., 2 (2010), no. 1, 75-83.

[68] V. Vougalter, V. Volpert. On the existence of stationary solutions for some non Fredholm integro-differential equations. Doc. Math., 16 (2011), 561-580.

[69] V. Volpert, V. Vougalter. Existence of stationary pulses for nonlocal reaction-diffusion equations, Doc. Math., 19 (2014), 1141-1153. 